\title{
An investigation into symptoms, diagnosis, treatment, and treatment complications in patients with retrosternal goiter.
}

\author{
Manouchehr Aghajanzadeh, Mohammad Reza Asgary*, Fereshteh Mohammadi, Haniye Darvishi, \\ Yasaman Safarpour \\ Inflammatory Lung Disease Research Center, Razi Hospital, School of Medicine, Guilan University of Medical \\ Science, Rasht, Iran
}

\begin{abstract}
Introduction: Retrosternal goiter refers to any thyroid enlargement in which over $50 \%$ of the thyroid permanently located under the thoracic inlet or the lower pole of thyroid is not palpable with the neck in hyperextended position. Due to the increasing number of surgical procedures of retrosternal goiter, the present study was carried out in order to examine the symptoms, diagnosis, treatment, and treatment complications in retrosternal goiter patients in Razi and Aria Hospitals, Rasht, Iran.

Material and methods: In this retrospective cross sectional descriptive study, between 2007 and 2013, from 777 patients who had undergone thyroidectomy, 70 patients $(9 \%)$ had retrosternal goiter. The medical records for 70 patients were retrospectively investigated. Data related to demographic, clinical symptoms, methods of diagnosis and postoperative complications were collected.

Results: According to the results of the present study, 71.4\% patients were women and most of the participants $(67.1 \%)$ aged 45 to $60 \mathrm{y}$. Mass in the neck was the most frequent symptoms before surgery (88.6\%). Total thyroidectomy was most common surgery $(\mathbf{7 4 . 3 \%})$. The most common incision for thyroidectomy $(95 / 7 \%)$ was neck collar incision. Diagnosis method in $82.9 \%$ and $17.1 \%$ of cases was respectively based on CT scans with CXR and CT scans with CXR and ultrasound. According to the postoperative pathologic findings, $\mathbf{5 8 . 5 \%}$ of the cases were multi-nodular goiter, $22.9 \%$ were papillary cell carcinoma, $7.1 \%$ were medullary carcinoma, $5.7 \%$ were anaplastic carcinoma, $5.7 \%$ were thyroid lymphoma, and only $1.4 \%$ were thyroid adenoma. Postoperative complications occurred in $47.14 \%$ of patients. Most common complication was early transient dysphonia. Permanent hypocalcemia were not observed in any patient.

Conclusion: This study recommends that retrosternal goiter should be operated early under suitable conditions, and the best diagnosis tool and best surgery methods are CT scan and surgery with collar incision, respectively.
\end{abstract}

Keywords: Surgery, Complications, Goiter, Retrosternal goiter.

Accepted on February 15, 2018

\section{Introduction}

Goiter is considered mediastinal if the lower pole of thyroid is not palpable with the neck in hyperextended position. Epidemiologically, it is difficult to have access to prevalence of retrosternal goiter. According to the reports; however, thyroid goiter develops into thorax in $2-21 \%$ of cases. In $7 \%$ of cases, retrosternal goiter appears as mediastinal tumor. Etiologically, $85-95 \%$ of retrosternal thyroid masses emerge as benign goiter. Histologically, goiter is created as a result of iodine deficiency. This problem is nowadays observed in developing countries. Goiter can also be due to consumption of goitrogenic substances which are substances that cause disruption in the production of thyroid hormones. Malignity happens in 5-15\% of retrosternal goiters.
Retrosternal goiters are more common among women. They develop slowly, their symptoms appear mostly in the $5^{\text {th }}$ and $6^{\text {th }}$ decades of life, and they usually put pressure on mediastinal structures. The most common symptoms of retrosternal goiter are dyspnea, respiratory problems, choking, and hoarseness.

Diagnosis is according to clinical symptoms and diagnostic methods like CXR and CT, and sometimes ultrasound also helps with diagnosis, but CT has high sensitivity. Surgery is the only effective method for retrosternal goiter. In most cases, suppressive therapies with levothyroxine are effective in reducing the size of multi-nodular goiter. Radioactive iodine is generally ineffective in decreasing goiter size and may cause acute inflammation, swelling of the glands, and an increase in the potential of airway obstruction. 
In most cases, surgery is carried out using a collar incision. Only $2 \%$ of patients who are considered for retrosternal goiter surgery may need another type of incision like manubriotomy, sternotomy, or thoracotomy.

Among the complications of retrosternal goiter surgery are hematoma, recurrent laryngeal nerve palsy, tracheomalasia, dysphonia, laryngeal edema, hypocalcemia, nausea, vomiting and wound complications after surgery, DVT, the need for transfusion after surgery due to the severity of bleeding, and mortality. In their study entitled, "Diagnosis, treatment, and surgery of retrosternal thyroid masses", Zhang and Cui examined 64 patients with retrosternal goiter [1].

These researchers reported that the best method to diagnose retrosternal goiter is using CXR and CT, and the most common surgery for this type of goiter is collar incision which is associated with minimum morbidity and recurrence rate [1].

By taking into account the surgery of 334 patients over 1993-2002, Aghajanzadeh et al. [2] stated that 32 patients had intrathoracic thyroid and were examined in a retrospective method in terms of diagnosis symptoms, treatment, and complications of the surgery. The results of that study showed that the most appropriate diagnosis tool is Chest X-ray (CXR) and the best tool is CT scan. Iodine thyroid scan was helpful in 5 cases. These researchers concluded that patients with retrosternal goiter need to undergo surgery during their treatment because they sometimes experience bleeding inside the goiter and malignant transformation, which is a serious threat [2].

An ever increase in the number of retrosternal goiter surgery on the one hand, and given the advances in new medical knowledge and diagnostic facilities on the other hand, it is expected that patient with retrosternal goiter are diagnosed more precisely and rapidly in order to reduce the complications caused by late diagnosis and delayed surgery and the financial costs imposed on society. The present study was carried out in order to precisely examine the records of the patients with retrosternal goiter who underwent surgery over 2007-2013, their symptoms, diagnosis method, and complications caused by the surgery.

\section{Materials and Methods}

In this retrospective cross sectional descriptive study, between 2007 and 2013, in Razi and Arya Hospitals, Rasht, Iran, from 777 patients that operated with thyroidectomy, 70 patients $(9 \%)$ had retrosternal goiter.

The study's variables included the frequency of the medication and the underlying diseases before hospitalization, symptoms before surgery, the conducted therapeutic methods, diagnosis methods (CXR, CT, ultrasonography and MRI), the postoperative pathologic findings, frequency distribution of the complications after the surgery according to the patients' sex, and the percentage of the complications based on age groups.

The measuring tool in the present study was a checklist that was based on individual information including age, sex, underlying diseases and the section of symptoms, diagnosistreatment methods, and the complications mentioned in the table of the variables. The patients' records were examined over the specified time, and there were no specific exclusion and inclusion criteria in the present study.

\section{Data collection instruments and method}

The records of all of the patients who were hospitalized for retrosternal goiter thyroidectomy were used in the present study. Then a questionnaire consisting of 2 sections of demographic information (age, sex, medication consumption, underlying disease, etc.) and clinical symptoms (bleeding, dyspnea, dysphagia, dysphonia, mass in the neck, hoarseness, asymptomatic), diagnosis method (CT, CXR, ultrasound, and MRI) and complications (early and late dysphonia, early and late dyspnea, transient and permanent recurrent laryngeal nerve injury, temporary and permanent hypocalcemia, and hoarseness) were completed for each patient.

\section{Data analysis method}

After the required data were collected, they were fed into SPSS 21.0 , frequency and confidence interval $95 \%$ were use in order to determine the symptoms, treatment-diagnosis method, and complications, and chi-square test was run to compare the data in terms of sex, age groups, and underlying diseases. The significance level was set at $5 \%$ for all tests.

\section{Ethical considerations}

Ethics and confidentiality were taken into account in extracting and recording the required data, and ethical consent letter was received from the patients to participate in the study.

\section{Results}

The results of the present study showed that out of 777 patients who had undergone thyroidectomy in Arya and Razi hospitals over 2007-2013, 70 patients had retrosternal goiter and out of whom $71.4 \%$ were women.

According to the results, most of the participants (46 people, $65.7 \%$ ) did not take any thyroid medication, $20.3 \%$ took levothyroxine, and $14 \%$ took methimazole. Examining underlying diseases before hospitalization showed that 46 patients $(65.7 \%)$ had euthyroid, 14 patients $(20.3 \%)$ had hypothyroid, and 10 patients (14\%) had hyperthyroid.

Mass in the neck was the most frequent symptoms before surgery $(88.6 \%)$. Total thyroidectomy and collar incision were the mostly used surgeries with respectively $74.3 \%$ and $95.7 \%$.

None of the participants received iodine therapy alone, and all of them underwent surgery. Diagnosis methods were CT scan along with chest X-ray in $82.9 \%$ of the cases and CT scan along with chest $\mathrm{X}$-ray and ultrasonography in $17.1 \%$ of the cases.

The comparative examination of frequency of postoperative complications according to the type of the data presented in 
Table 1 shows that $60 \%$ of the men (12 patients) and $42 \%$ of the women (21 patients) experienced postoperative complications; however, this difference was not significant although there was a difference of $18 \%$ based on chi-square test. In comparative examining the percentage of the complications based on age groups, presented in Table 2 , it was shown that the percentage of the complications among individuals under 45 y was $38.5 \%$, among those between 45 and $60 \mathrm{y}$ was $44.7 \%$, and among those over $60 \mathrm{y}$ was $70 \%$. Although the difference between the age groups was over $30 \%$, this difference was not significant due to lack of participants in age group of over 60 years (Table 2).

Table 1. Comparative examining of postoperative complications percentage according to age and sex groups.

\begin{tabular}{|c|c|c|c|c|c|}
\hline & & \multicolumn{2}{|c|}{$\begin{array}{l}\text { Postoperative } \\
\text { complications }\end{array}$} & \multirow[t]{2}{*}{ Total } & \multirow[t]{2}{*}{$\mathbf{P}$} \\
\hline & & Yes & No & & \\
\hline \multirow[t]{5}{*}{ Sex } & Count & 12 & 8 & 20 & 0.173 \\
\hline & Male $\%$ within sex & $60 \%$ & $40 \%$ & $100 \%$ & \\
\hline & Count & 21 & 29 & 50 & \\
\hline & Female $\%$ within sex & $42 \%$ & $58 \%$ & $100 \%$ & \\
\hline & Count & 33 & 37 & 70 & \\
\hline Total & $\%$ within sex & $47.10 \%$ & $52.90 \%$ & $100 \%$ & \\
\hline
\end{tabular}

Table 2. Comparing the percentage of postoperative complications according to age groups among patients with retrosternal goiter.

\begin{tabular}{|c|c|c|c|c|c|}
\hline & & \multicolumn{2}{|c|}{$\begin{array}{l}\text { Postoperative } \\
\text { complications }\end{array}$} & \multirow[t]{2}{*}{ Total } & \multirow[t]{2}{*}{$\mathbf{P}$} \\
\hline & & Yes & No & & \\
\hline \multirow[t]{8}{*}{ Age group } & Count & 5 & 8 & 13 & 0.272 \\
\hline & $\begin{array}{l}\text { Blow } 45 \text { y } \% \text { within age } \\
\text { group }\end{array}$ & $38.50 \%$ & $61.50 \%$ & $100 \%$ & \\
\hline & Count & 21 & 26 & 47 & \\
\hline & $\begin{array}{l}45 \text { to } 60 \text { y } \% \text { within age } \\
\text { group }\end{array}$ & $44.70 \%$ & $55.30 \%$ & $100 \%$ & \\
\hline & Count & 7 & 3 & 10 & \\
\hline & $\begin{array}{l}\text { Over } 60 \text { y } \% \text { within age } \\
\text { group }\end{array}$ & $70 \%$ & $30 \%$ & $100 \%$ & \\
\hline & Count & 33 & 37 & 70 & \\
\hline & Total $\%$ within age group & $47.10 \%$ & $52.90 \%$ & $100 \%$ & \\
\hline
\end{tabular}

In examining the postoperative pathologic findings, most of the participants (41 patients, $58.5 \%$ ) were reported to have multinodular goiter, $22.9 \%$ (16 patients) papillary cell carcinoma, $7.1 \%$ (5 patients) medullary carcinoma, 5.7\% (4 patients) anaplastic carcinoma, $5.7 \%$ (4 patients) thyroid lymphoma, and only $1.4 \%$ ( 1 patient) thyroid adenoma (Table 3 ).

Table 3. Examining the postoperative pathologic findings (lymphoma, anaplastic, medullary, etc.) among the patients.

\begin{tabular}{llll}
\hline & MNG & 41 & $58.50 \%$ \\
\hline $\begin{array}{l}\text { Postoperative pathologic findings } \\
\text { (lymphoma, anaplastic, medullary, }\end{array}$ & PTC & 16 & $22.90 \%$ \\
\cline { 2 - 4 } etc.) & Medullary & 5 & $7.10 \%$ \\
\cline { 2 - 4 } & Anaplastic & 4 & $5.70 \%$ \\
\cline { 2 - 4 } & Lymphoma & 4 & $5.70 \%$ \\
\cline { 2 - 4 } & Total & 70 & $100 \%$ \\
\hline
\end{tabular}

\section{Discussion}

Retrosternal goiter was first described by Haller in 1794. It prevalence varies 3-20\% among patients who undergo thyroid surgery. Its extensive prevalence is due to different definitions of this type of goiter [3]. In the present study, prevalence of Retrosternal goiter was 9\%.

In the present study, most of the participants (88.6\%) had neck mass, 70\% (49 patients) had dyspnea, 27.1\% (19 patients) had dysphonia, 22.9\% (16 patients) had dysphagia, 15.7\% (11 patients) had hoarseness, and only 8 patients (11.4\%) had no symptoms before surgery. In a study carried out in 2006, dyspnea was reported as the most common functional symptom among young patients $(39.3 \%)$ due to compression of the trachea by the goiter, and dysphagia $(16.2 \%)$ and the second most common symptom that has no relationship with goiter development among this group of patients. In their study, Grainger et al. [4] reported that patients with large goiter glands can have no symptoms in $10-35 \%$ of cases, which is similar to the present study. According to the results of the study carried out by Siderys, $17-32 \%$ of the patients who undergo surgery are asymptomatic, and their goiter is accidentally diagnosed in their chest X-ray. In these patients, due to consequent hazardous complications, it is better to treat goiter by prophylaxis method [5]. In the present study, diagnosis methods were CT scan along with chest X-ray in $82.9 \%$ of the cases and CT scan along with chest $\mathrm{X}$-ray and ultrasonography in $17.1 \%$ of the cases.

The results of the present study indicated that $100 \%$ of the findings obtained from chest $\mathrm{x}$-ray had tracheal deviation, and in $100 \%$ of the findings obtained from CT scan, there were superior mediastinal mass, tracheal deviation and tracheal stenosis. Ultrasonography was done for $17.1 \%$ of the patients, and 100 of them had multi-nodular goiter in both thyroid lobes and larger-than-normal thyroid. MRI was not carried out for any of the patients. In the study carried out by Cui et al. the highest rate of diagnosis was related to CXR (70\%) and CT scan (96\%) [1]. Chest X-ray (CXR) is abnormal in most cases [6]. Studies indicated that radiography plays a limited role in measuring thyroid diseases. Radiography can show soft tissue masses, tracheal deviation, and calcifications in thyroid. Radiography findings; however, have neither sensitivity nor specificity. As tracheal deviation and stenosis can be due to another reason except for retrosternal goiter, calcification in neck can be due to several other reasons except for thyroid cancer, and lung and bone metastases can be originated from several primary places. Like ultrasonography, MRI is not a 
sensitive method to show lesions in the thyroid, and does not have enough specificity to not prefer other imaging diagnosis methods over it. It is; however, useful in lymphadenopathy evaluation and development of thyroid neoplasms. Nowadays, MRI plays a limited role in diagnosing thyroid nodules. It seems to be useful in diagnosis of neck lymph node metastasis. CT scan is a useful method to evaluate lymphadenopathy, local development of thyroid, and spread to mediastinum or retrotracheal zone. Ultrasonography is an appropriate method for primary diagnosis of lesions in the thyroid, and CT scan and MRI are useful to stage the lesions. However, it cannot distinguish benign and malignant nodules [7]. Neck scan is not the routine part of estimating thyroid nodule, but it can be used in patients with significant pressure or evaluating the retrosternal area or tracheal density. In the present study, $25 \%$ of the participants underwent FNA before the surgery, and their pathological findings indicated 2 cases of modular goiter and 6 cases of multi-nodular goiter. In the present study, $74.3 \%$ of the patients underwent total thyroidectomy, $25.7 \%$ subtotal thyroidectomy, $95.7 \%$ collar incision, and $4.3 \%$ median sternotomy. In their study, Gourin et al reported that the most common retrosternal goiter surgery is neck incision, and sternotomy is carried out in rare cases, which is similar to the present study. However, selecting surgery method is completely dependent on the surgeon's experience and a method is chosen that led to minimum threat in terms of bleeding and hematoma [8].

Available data on treatment methods for patients with retrosternal goiter indicated that almost all of the patients underwent surgery, and only 2 patients $(2.9 \%)$ had surgery along with iodine therapy before surgery, and none of them had iodine therapy alone. Radioisotope iodine 123 is often a choice that indicates metastatic cancer well, and one third of papillary carcinoma and two third of follicular carcinoma are so iodine absorbent that they can be seen. But, radionucleotide imaging is not reliable in approve or reject cancer [7]. Moreover, using radioactive iodine to diagnose retrosternal goiter before surgery may lead to dyspnea, cause severe inflammation in airways, and make the disease an emergency condition which should be dealt with much care [9].

In the present study, totally $47.14 \%$ of the participants experienced complications after the surgery, but $52.9 \%$ had no complications. Among the complications, $25.7 \%$ of the participants had early dysphonia, $12.9 \%$ had early dyspnea, $11.4 \%$ had transient hypocalcemia, $2.9 \%$ had late dysphonia, and $7.1 \%$ had postoperative bleeding. Permanent hypocalcemia was not seen in any of the patients. The results of a study carried out on 75 patients by Ben et al. [10] showed that transient recurrent nerve injury after surgery occurred in $7 \%$ of the patients, permanent nerve damage in $4 \%$ of the patients, transient hypocalcemia in $10 \%$ of them, and permanent hypocalcemia in $2.6 \%$ of them.

Examining the postoperative pathologic findings showed that $58.5 \%$ of the cases were multi-nodular goiter, $22.9 \%$ were papillary cell carcinoma, $7.1 \%$ were medullary carcinoma, $5.7 \%$ were anaplastic carcinoma, $5.7 \%$ were thyroid lymphoma, and only $1.4 \%$ were thyroid adenoma. In a study conducted in 2002, postoperative pathologic findings showed that most cases were multi-nodular goiter $(54.7 \%)$, thyroid adenoma (21.9\%), and thyroid carcinoma (15.6\%) [1]. In a study, Aghajanzadeh [2] reported that $68.8 \%$ of the patients had multi-nodular goiter, $21.9 \%$ had papillary cell carcinoma, $3.1 \%$ had modular cell carcinoma, 3.12\% had anaplastic carcinoma, and $3.1 \%$ had thyroid lymphoma. Retrosternal goiter patients' precaution is highly significant. Chest goiter along with papillary and medullary cancers had highly acceptable results and good survival rate [11]. In retrosternal goiter; however, patients with anaplastic and lymphoma cancers had a survival rate of about 6 month to 1 year.

\section{Conclusion}

The results of the present study showed that incidence of retrosternal goiter are higher among women. Mass in the neck was the most frequent symptoms before surgery. In our study, the possibility of cancer was high $(41.5 \%)$. Bleeding in goiter leads to high compression on the trachea and creation of an emergency condition; therefore, it is better for all patients to undergo surgery in appropriate conditions unless there are cardiovascular problems.

\section{Conflicts of Interest}

The authors declare no conflict of interest

\section{References}

1. Cui YZZ. Diagnosis and surgical management for the retrosternal thyroid mass. Chin Med Sci J 2002; 17: 173-177.

2. Aghajanzadeh M, Safarpour F, Kouhsari M, Alavi S, Amani H, Kalantari S. Substernal goiters: clinical presentation, diagnosis, and surgical management. Iran J Endicrinol Metabol 2007.

3. Wright DMD. Mediastinal tumors: diagnosis and treatment. World J Surg 2001; 25: 204-209.

4. Gonçalves JKL. Surgical complications after thyroid surgery performed in a cancer hospital. Otolaryngol Head Neck Surg 2005; 132: 490-494.

5. Siderys HRG. Superior vena caval syndrome caused by intrathoracic goiter. Am J Surg 1970; 36: 446-450.

6. Shaha AR BC, Alfonso A, Jaffe BM. Goiters and airway problems. Am J Surg 2010; 158: 378-380.

7. Ket S, Ozbudak O, Ozdemir T, Dertsiz L. Acute respiratory failure and tracheal obstruction in patients with posterior giant mediastinal (intrathoracic) goiter. Interact Cardiovasc Thorac Surg 2004; 3: 174-175.

8. Gourin AGA, Karlson KE. The cervicomediastinal approach to intrathoracic goiter. Surgery 1971; 69: 651-654.

9. Park HMTR, Siddiqui AR, Schauwecker DS, Wellman $\mathrm{HN}$, Efficacy of thyroid scintigraphy in the diagnosis of intrathoracic goiter. Am J Roentgenol 1987; 148: 527-529. 
An investigation into symptoms, diagnosis, treatment, and treatment complications in patients with retrosternal goiter

10. Ayache sMN, Tramier B, Strunski V. Clinical signs correlation with radiological extent in a series of 117 retrosternal goiter. Revue de Laryngologie-OtologieRhinologie 2006; 127: 229-237.

11. Katlic MRGH, Wang CA. Substernal goiter. Analysis of 80 patients from Massachusetts General Hospital. Am J Surg 2006; 194: 283-287.

\section{*Correspondence to}

Mohammad Reza Asgary
Inflammatory Lung Disease Research Center

Razi Hospital

School of Medicine

Guilan University of Medical Science

Iran 